\title{
Association between somatic RET mutations and clinical and genetic characteristics in patients with metastatic colorectal cancer
}

\section{Yuan-Zhong Yang}

Department of Pathology, Sun Yat-sen University Cancer Center; State Key Laboratory of Oncology in South China

\section{Wan-Ming Hu}

Department of Pathology, Sun Yat-sen University Cancer Center; State Key Laboratory of Oncology in South China

\section{Liang-Ping Xia}

VIP Region, Sun Yat-sen University Cancer Center; State Key Laboratory of Oncology in South China wen-zhuo He ( $\nabla$ hewzh@sysucc.org.cn )

Sun Yat-sen University Cancer Center https://orcid.org/0000-0002-7423-4999

\section{Research}

Keywords: colorectal cancer, RET, mucinous histology, peritoneal metastasis

Posted Date: January 20th, 2021

DOl: https://doi.org/10.21203/rs.3.rs-150168/v1

License: (c) (1) This work is licensed under a Creative Commons Attribution 4.0 International License. Read Full License

Version of Record: A version of this preprint was published at Cancer Medicine on November 5th, 2021. See the published version at https://doi.org/10.1002/cam4.4400. 


\section{Abstract \\ Background}

Rearranged during transfection (RET) is a targetable oncogene. RET fusions have been reported in patients with metastatic colorectal cancer (mCRC). However, RET mutations in $\mathrm{mCRC}$ are less studied. Here, we aimed to characterize the clinical, pathological, and molecular landscape of RET-mutated mCRC.

\section{Methods}

Five hundred and eighty-two patients were included in this study. Next-generation sequencing was performed to detect RET mutations and calculate tumor mutation burden (TMB). We compared the clinical, pathological, and molecular characteristics of $\mathrm{mCRC}$ cases with tumors that harbored somatic $R E T$ mutations $(N=16,2.7 \%)$ or had wild-type $R E T(N=566,97.3 \%)$.

\section{Results}

Males comprised the absolute majority of cases with RET mutations (15/16 [93.8\%]) compared to their fraction among cases with wild-type RET (339/566 [59.9\%]). Furthermore, all patients with RET mutations were younger than 60 years $(16 / 16[100 \%])$, whereas such patients were less predominant in the group with wild-type $R E T$ (379/566 [67.0\%]). Individuals with tumors positive for $R E T$ mutations more frequently exhibited mucinous histology (5/16 [31.2\%] versus 55/566 [9.7\%]), exhibited a lower incidence of liver metastasis (4/16 [25.0\%] versus 335/566 [59.2\%]) and higher incidence of peritoneal metastasis $(9 / 16$ [56.2\%] versus 161/566 [28.4\%]), expressed wild-type TP53 (8/16 [50.0\%] versus 120/566 [21.2\%]), and showed an increased frequency of MSI-high (6/16 [37.5\%] versus 18/566 [3.2\%]). In those with microsatellite-stable $\mathrm{MCRC}$, patients with RET mutations had a higher median TMB than patients with wild-type $R E T$ (9.4 versus 6.7 mutations/Mb, respectively, $P=0.001$ ). The median progression-free survival was similar in individuals with mutated and wild-type $R E T$ on the oxaliplatin-based regimen (7.1 versus 8.7 months, $P=0.516$ ).

\section{Conclusions}

Our study suggests that cases with RET mutations represent a separate mCRC subtype. Further studies are needed to evaluate the efficacy of RET inhibitors in MCRC patients with RET mutations.

\section{Background}

The RET (rearranged during transfection) gene encodes a receptor tyrosine kinase that plays a key role in the activation of MAPK, PI3K, JAK, and PKA/C pathways [1]. Aberrant RET activation is involved in various types of tumorigenesis, including medullary thyroid cancer (MTC), non-small cell lung cancer 
(NSCLC), and colorectal cancer (CRC) [2-4]. Thus, RET has emerged as a therapeutic target in patients with aberrant RET activation. Two selective RET inhibitors, selpercatinib (LOXO-292) and pralsetinib (BLU667), have shown promising anti-cancer activities and are currently widely evaluated in clinical trials [2, 5-8].

There are mainly two mechanisms underlying RET oncogenic activation. The first is chromosomal rearrangement, which causes fusion of RET with a partner protein $[9,10]$. The second mechanism is somatic or germline gain-of-function mutation [11]. RET fusions were observed in a small fraction $(<1 \%)$ of metastatic $\mathrm{CRC}$ ( $\mathrm{mCRC}$ ), where their presence was associated with old age, right-sided tumor origin, wild-type RAS and BRAF, microsatellite instability (MSI)-high tumors, and poor prognosis [12]. However, the prevalence as well as clinical, pathological, and molecular features of somatic RET mutations in patients with $\mathrm{mCRC}$ are largely unknown. Kato et al. examined a cohort of 300 patients with CRC and identified two individuals with RET mutations [13]. Such rare occurrence of $R E T$ mutations precluded their further analysis. Hence, this study aimed to evaluate the frequency and phenotypic characteristics of mCRC with somatic RET mutation in a larger patient cohort.

\section{Methods}

The following patients were selected: (1) those diagnosed with mCRC based on pathology samples at our institute between January 1, 2015 and March 31, 2020; (2) those who had an available record of the nextgeneration sequencing result performed at our institute. Those who (1) accepted chemotherapy or radiotherapy before next-generation sequencing analysis or (2) had no available follow-up information were excluded. The study was approved by the Institutional Review Board of Sun Yat-sen University Cancer Center, and all patients provided informed consent. The detailed description of the methods for next-generation sequencing and tumor mutation burden (TMB) calculation is provided in the Supplementary Material.

We evaluated the association between the presence of somatic RET mutations and the following variables: age, gender, primary tumor location, differentiation, mucinous histology, $\mathrm{T}$ stage, $\mathrm{N}$ stage, time to metastasis, metastatic organs, TP53 mutations, APC mutation, RAS mutations, BRAF mutations, SMAD4 mutations, PIK3CA mutations, ERBB2 amplifications, and MSI status. Patients' characteristics were compared by using the chi-squared test. TMBs were compared by using the Kruskal-Wallis test. Kaplan-Meier survival analysis and log-rank test were performed to detect differences in progression-free survival (PFS). The statistical tests were two-tailed and the differences were considered statistically significant when $P<0.05$.

\section{Results}

Five hundred and eighty-two patients were included in this study (Fig. 1). The patients' median age was 54 (range, 17-88) years, and 354 patients (60.8\%) were male. Somatic RET mutations were identified in 16 (2.7\%) patients. The genomic alterations of RET-mutated tumors are shown in Table 1 and Fig. 2. 
Table 1

Genomic Alterations in RET-Mutated Metastatic Colorectal Cancer

\begin{tabular}{|c|c|}
\hline Patient ID & Genomic alterations \\
\hline 1 & $R E T, \mathrm{EX} 3, \mathrm{R} 177 \mathrm{~W} ; R A S$ wild-type; $B R A F$ wild-type; MSS \\
\hline 2 & RET, EX3, R133C; KRASA146T; BRAF wild-type; MSS \\
\hline 3 & $R E T$, EX5, V351l; RAS wild-type; $B R A F$ wild-type; MSI-high \\
\hline 4 & $R E T$, EX6, N359T; RAS wild-type; BRAF wild-type; MSS \\
\hline 5 & RET, EX7, T451M; KRAS G12D; BRAF wild-type; MSS \\
\hline 6 & RET, EX7, V485L; NRASQ61L; BRAF wild-type; MSI-high \\
\hline 7 & RET, EX10, E616del; KRAS G12D; BRAF wild-type; MSI-high \\
\hline 8 & RET, EX10, E623G; KRAS G13D; BRAF wild-type; MSS \\
\hline 9 & $R E T$, EX11, V642F; RAS wild-type; BRAF wild-type; MSS \\
\hline 10 & $R E T$, EX11, K662M; RAS wild-type; BRAF wild-type; MSS \\
\hline 11 & RET, EX11, P695S; KRAS G13D; BRAF wild-type; MSI-high \\
\hline 12 & $R E T$, EX11, V706M; RAS wild-type; BRAF wild-type; MSS \\
\hline 13 & $R E T$, EX15, V871l; RAS wild-type; BRAF wild-type; MSI-high \\
\hline 14 & $R E T$, EX15, V871l; KRAS G13D; BRAF wild-type; MSI-high \\
\hline 15 & $R E T$, EX15, S891L; RAS wild-type; BRAF wild-type; MSS \\
\hline 16 & RET, EX15, S891L; RAS wild-type; BRAF K601E; MSS \\
\hline
\end{tabular}

The presence of $R E T$ mutations was associated with male sex (15/16 [93.8\%] patients with mutated RET vs. 339/566 [59.9\%] patients with wild-type RET), younger age (all 16 patients with mutated $R E T$ [100\%] were $\leq 60$ years old vs. 379 out of $566[67.0 \%]$ patients with wild-type RET), mucinous histology (5/16 [31.2\%] patients with mutated RET vs. 55/566 [9.7\%] patients with wild-type RET), less liver metastasis (4/16 [25.0\%] patients with mutated RET vs. 335/566 [59.2\%] patients with wild-type RET), and more peritoneal metastasis (9/16 [56.2\%] patients with mutated RET vs. $161 / 566$ [28.4\%] patients with wildtype $R E T$, Table 2). Nine (12.3\%) out of 73 male patients who were younger than 60 years of age and had peritoneal metastasis harbored RET mutations. 
Table 2

Characteristics of Patients with RET-Mutated Metastatic Colorectal Cancer

\begin{tabular}{|c|c|c|c|}
\hline Characteristics & $R E T^{\text {mutation }}, N(\%)$ & $R E T$ wild-type,$N(\%)$ & $P$ \\
\hline Gender & & & 0.007 \\
\hline Male & $15(93.8)$ & 339 (59.9) & \\
\hline Female & $1(6.2)$ & $227(40.1)$ & \\
\hline Age & & & 0.002 \\
\hline$>60$ & $0(0)$ & $187(33.0)$ & \\
\hline$\leq 60$ & $16(100)$ & $379(67.0)$ & \\
\hline Primary tumor location & & & 0.603 \\
\hline Right colon & $6(37.5)$ & $162(28.6)$ & \\
\hline Left colon & $6(37.5)$ & $198(35.0)$ & \\
\hline Rectum & $4(25.0)$ & $206(36.4)$ & \\
\hline Differentiation & & & 0.784 \\
\hline Well / moderate & $11(68.8)$ & $416(73.5)$ & \\
\hline Poor & $5(31.2)$ & $150(26.5)$ & \\
\hline Mucinous histology & & & 0.018 \\
\hline Yes & $5(31.2)$ & $55(9.7)$ & \\
\hline No & $11(68.8)$ & $511(90.3)$ & \\
\hline T stage & & & 0.415 \\
\hline 1 & $0(0)$ & $2(0.4)$ & \\
\hline 2 & $0(0)$ & $21(3.7)$ & \\
\hline 3 & $5(31.2)$ & $266(47.0)$ & \\
\hline 4 & $7(43.8)$ & $161(28.4)$ & \\
\hline N stage & & & 0.029 \\
\hline 0 & $6(37.5)$ & $123(21.7)$ & \\
\hline 1 & $0(0)$ & $164(29.0)$ & \\
\hline 2 & $5(31.2)$ & $146(25.8)$ & \\
\hline Time to metastasis & & & 0.269 \\
\hline
\end{tabular}




\begin{tabular}{|c|c|c|c|}
\hline Characteristics & $R E T^{\text {mutation }}, N(\%)$ & $R E T$ wild-type,$N(\%)$ & $P$ \\
\hline Synchronous & $9(56.2)$ & $398(70.3)$ & \\
\hline Metachronous & $7(43.8)$ & $168(29.7)$ & \\
\hline Liver metastasis & & & 0.009 \\
\hline Yes & $4(25.0)$ & $335(59.2)$ & \\
\hline No & $12(75.0)$ & $231(40.8)$ & \\
\hline Lung metastasis & & & 0.257 \\
\hline Yes & $2(12.5)$ & $159(28.1)$ & \\
\hline No & $14(87.5)$ & 407 (71.9) & \\
\hline Peritoneal metastasis & & & 0.024 \\
\hline Yes & $9(56.2)$ & $161(28.4)$ & \\
\hline No & $7(43.8)$ & $405(71.6)$ & \\
\hline Distant nodes metastasis & & & 0.452 \\
\hline Yes & $3(18.8)$ & 73 (12.9) & \\
\hline No & $13(81.2)$ & $493(87.1)$ & \\
\hline
\end{tabular}

As shown in Table 3, RET mutations were more frequent in patients with wild-type TP53 and MSI-high tumors (6/16 [37.5\%] patients with mutated RET vs. 18/566 [3.2\%] patients with wild-type RET). There were no significant associations between RET mutation presence and APC, RAS, BRAF, SMAD4, PIK3CA, or ERBB2 status. In patients with microsatellite-stable $\mathrm{mCRC}$, those with $R E T$ mutations had a higher TMB than those with wild-type RET (median TMB 9.4 and 6.7 Muts/Mb respectively, $P=0.001$ ). In patients with MSI-high mCRC, those with RET mutations had a TMB similar to that of patients with wildtype RET (median TMB 66.6 and 59.2 Muts/Mb, respectively, $P=0.923$ ). 
Table 3

Association between the Presence of RET Mutation and Other Molecular Characteristics

\begin{tabular}{|c|c|c|c|}
\hline Characteristics & $R E T^{\text {mutation }}, N(\%)$ & $R E T^{\text {wild-type }}, N(\%)$ & $P$ \\
\hline TP53 & & & 0.012 \\
\hline Wild-type & $8(50.0)$ & $120(21.2)$ & \\
\hline Mutated & $8(50.0)$ & $446(78.8)$ & \\
\hline$A P C$ & & & 0.595 \\
\hline Wild-type & $4(25.0)$ & $194(34.3)$ & \\
\hline Mutated & $12(75.0)$ & $372(65.7)$ & \\
\hline$R A S$ & & & 0.622 \\
\hline Wild-type & $9(56.2)$ & $280(49.5)$ & \\
\hline Mutated & $7(43.8)$ & $286(50.5)$ & \\
\hline$B R A F$ & & & 0.319 \\
\hline Wild-type & $15(93.8)$ & 503 (88.9) & \\
\hline V600E mutation & $0(0)$ & $49(8.7)$ & \\
\hline No V600E mutation & $1(6.2)$ & $14(2.4)$ & \\
\hline SMAD4 & & & 0.500 \\
\hline Wild-type & $12(75.0)$ & $468(82.7)$ & \\
\hline Mutated & $4(25.0)$ & 98 (17.3) & \\
\hline PIKЗCA & & & 0.721 \\
\hline Wild-type & $13(81.2)$ & $481(85.0)$ & \\
\hline Mutated & $3(18.8)$ & $85(15.0)$ & \\
\hline ERBB2 & & & 1.000 \\
\hline Amplification & $16(100)$ & $548(96.8)$ & \\
\hline No amplification & $0(0)$ & $18(3.2)$ & \\
\hline MSI status & & & $<0.001$ \\
\hline MSS & $10(62.5)$ & $548(96.8)$ & \\
\hline MSI-high & $6(37.5)$ & $18(3.2)$ & \\
\hline
\end{tabular}


The majority of the studied patients $(413 / 582,71.0 \%)$ were administered oxaliplatin-based first-line chemotherapy (FOLFOX or XELOX), whereas 63 (10.8\%) received irinotecan-based chemotherapy (FOLFIRI or XELIRI). Among the mCRC patients on the oxaliplatin-based regimen, PFS was similar between patients with RET mutations and those with wild-type RET (median PFS 7.1 vs. 8.7 months, respectively, $P=0.516$, Fig. 3).

\section{Discussion}

In our analysis of 582 patients with mCRC, we detected RET mutations in $16(2.7 \%)$ individuals. Furthermore, $R E T$ mutations were more often present in younger male patients and they were associated more strongly with mucinous histology, weaker liver metastasis, and stronger peritoneal metastasis. Given that the incidence of RET mutations in mCRC patients is low, our study provides valuable information to potentially help physicians identify the enriched subgroups in which RET mutations could be more frequent.

Kato et al. studied RET aberrations in 4,871 patients with diverse malignancies [13]. Three hundred patients with CRC were included, and only two of them $(0.7 \%)$ had $R E T$ mutations. The tumor stages were unknown, and this might explain the low mutation rate of $R E T$ in that study. Recently, Zhao et al. retrospectively reviewed 3,272 patients with CRC and documented that the RET mutation rate was 3.39\% [11], which was similar to the rate observed in the present study. The additional information regarding the association of RET mutations with other factors (sex, age, histology, and metastasis sites) revealed in our study suggests a potential enrichment strategy for further trials with targeted agents focused on the RET mutation.

To date, there are several commercially available inhibitors demonstrating activity against RET. Most of them are non-specific inhibitors, such as cabozantinib, vandetanib, lenvatinib, sorafenib, sunitinib, and alectinib [14]. Recently, two selective RET inhibitors have been introduced to the clinic. Pralsetinib has been demonstrated to inhibit the growth of tumors driven by various RET mutations and fusions in vivo, and it showed durable clinical responses in patients with RET-altered NSCLC $[15,16]$. Selpercatinib also showed promising activity in patients with RET-fused NSCLC and RET-fused or mutated MTC [7, 8, 14, 17]. Two patients with RET-mutated mCRC were included in a clinical trial (NCT03037385), and the efficacy of pralsetinib is still under evaluation in these two patients.

RET fusions have been observed in a small subgroup of patients with mCRC. RET fusions were associated with older age, origin on the right side, wild-type $R A S$ and $B R A F$, MSI-high tumors, as well as shorter survival [12]. In this study, RET mutations were enriched in younger and male patients. We did not observe significant correlations between the presence of $R E T$ mutations on one hand and primary tumor location and RAS or BRAF status on the other hand. These results suggest that factors affecting the incidence of $R E T$ mutations are different from those associated with RET rearrangements.

Our study had few limitations, such as a limited sample size and retrospective bias. The identified RET mutations in this study could be driver aberrations or only neutral passengers. We could not distinguish 
between them owing to limited information regarding $R E T$ mutations in mCRC. Furthermore, because the follow-up time was limited, overall survival could not be determined.

\section{Conclusions}

Despite these limitations, our study suggests that tumors with $R E T$ mutation represent a novel subtype of mCRC. Further studies are needed to evaluate the efficacy of RET inhibitors in patients with RET-mutated mCRC.

\section{Abbreviations}

RET

rearranged during transfection

MTC

medullary thyroid cancer

NSCLC

non-small cell lung cancer

CRC

colorectal cancer

mCRC

metastatic colorectal cancer

MSI

microsatellite instability

TMB

tumor mutation burden

PFS

progression-free survival

\section{Declarations}

Ethics approval and consent to participate:

The study was approved by the Institutional Review Board of Sun Yat-sen University Cancer Center (GZKJ2020-023).

Consent for publication:

Not applicable.

Availability of data and materials: 
The datasets analyzed during the current study are available in the Research Data Deposit system of Sun Yat-sen University Cancer.

\section{Competing interests:}

The authors declare that they have no competing interests

\section{Funding:}

This work was supported by grants from the Guangdong Medical Science and Technology Research Fund (C2018063).

\section{Authors' contributions}

Concept and design: All authors.

Acquisition, analysis, or interpretation of data: Yuan-Zhong Yang and Wan-Ming Hu.

Drafting of the manuscript: Wen-Zhuo He and Liang-Ping Xia.

Critical revision of the manuscript for important intellectual content: All authors.

Statistical analysis: Wen-Zhuo He, Wan-Ming Hu and Liang-Ping Xia.

Obtained funding: Wen-Zhuo He.

Administrative, technical, or material support: Yuan-Zhong Yang and Wan-Ming Hu.

Supervision: Liang-Ping Xia.

\section{Acknowledgements}

We have engaged the services of Editage [www.editage.cn], an English language editing service, to improve language in the manuscript.

\section{References}

1. Kohno T, Tabata J, Nakaoku T: REToma: a cancer subtype with a shared driver oncogene. Carcinogenesis 2020, 41(2):123-129.

2. Belli C, Anand S, Gainor JF, Penault-Llorca F, Subbiah V, Drilon A, Andre F, Curigliano G: Progresses Toward Precision Medicine in RET-altered Solid Tumors. Clin Cancer Res 2020, 26(23):6102-6111.

3. Romei C, Ciampi R, Elisei R: A comprehensive overview of the role of the RET proto-oncogene in thyroid carcinoma. Nat Rev Endocrinol 2016, 12(4):192-202.

4. Mulligan LM: RET revisited: expanding the oncogenic portfolio. Nat Rev Cancer 2014, 14(3):173-186. 
5. Subbiah V, Gainor JF, Rahal R, Brubaker JD, Kim JL, Maynard M, Hu W, Cao Q, Sheets MP, Wilson D et al: Precision Targeted Therapy with BLU-667 for RET-Driven Cancers. Cancer Discov 2018, 8(7):836849.

6. Drilon A, Hu ZI, Lai GGY, Tan DSW: Targeting RET-driven cancers: lessons from evolving preclinical and clinical landscapes. Nat Rev Clin Oncol 2018, 15(3):151-167.

7. Drilon A, Oxnard GR, Tan DSW, Loong HHF, Johnson M, Gainor J, McCoach CE, Gautschi O, Besse B, Cho BC et al: Efficacy of Selpercatinib in RET Fusion-Positive Non-Small-Cell Lung Cancer. N Engl J Med 2020, 383(9):813-824.

8. Bradford D, Larkins E, Mushti SL, Rodriguez L, Skinner AM, Helms WS, Price LSL, Fourie Zirkelbach J, Li Y, Liu J et al: FDA Approval Summary: Selpercatinib for the Treatment of Lung and Thyroid cancers with RET Gene Mutations or Fusions. Clin Cancer Res 2020.

9. Li J, Shang G, Chen YJ, Brautigam CA, Liou J, Zhang X, Bai XC: Cryo-EM analyses reveal the common mechanism and diversification in the activation of RET by different ligands. Elife 2019, 8.

10. Bigalke JM, Aibara S, Roth R, Dahl G, Gordon E, Dorbeus S, Amunts A, Sandmark J: Cryo-EM structure of the activated RET signaling complex reveals the importance of its cysteine-rich domain. Sci Adv 2019, 5(7):eaau4202.

11. Zhao Z, Fu T, Gao J, Xu Y, Wu X, Chen W, Li X, Yu R, Shao YW, Li M et al: Identifying novel oncogenic RET mutations and characterising their sensitivity to RET-specific inhibitors. J Med Genet 2020.

12. Pietrantonio F, Di Nicolantonio F, Schrock AB, Lee J, Morano F, Fuca G, Nikolinakos P, Drilon A, Hechtman JF, Christiansen $\mathrm{J}$ et al: RET fusions in a small subset of advanced colorectal cancers at risk of being neglected. Ann Oncol 2018, 29(6):1394-1401.

13. Kato S, Subbiah V, Marchlik E, Elkin SK, Carter JL, Kurzrock R: RET Aberrations in Diverse Cancers: Next-Generation Sequencing of 4,871 Patients. Clin Cancer Res 2017, 23(8):1988-1997.

14. Subbiah V, Yang D, Velcheti V, Drilon A, Meric-Bernstam F: State-of-the-Art Strategies for Targeting RET-Dependent Cancers. J Clin Oncol 2020, 38(11):1209-1221.

15. Subbiah V, Cote GJ: Advances in Targeting RET-Dependent Cancers. Cancer Discov 2020, 10(4):498505.

16. Stinchcombe TE: Current management of RET rearranged non-small cell lung cancer. Ther Adv Med Oncol 2020, 12:1758835920928634.

17. Wirth LJ, Sherman E, Robinson B, Solomon B, Kang H, Lorch J, Worden F, Brose M, Patel J, Leboulleux S et al: Efficacy of Selpercatinib in RET-Altered Thyroid Cancers. N Engl J Med 2020, 383(9):825-835.

\section{Figures}




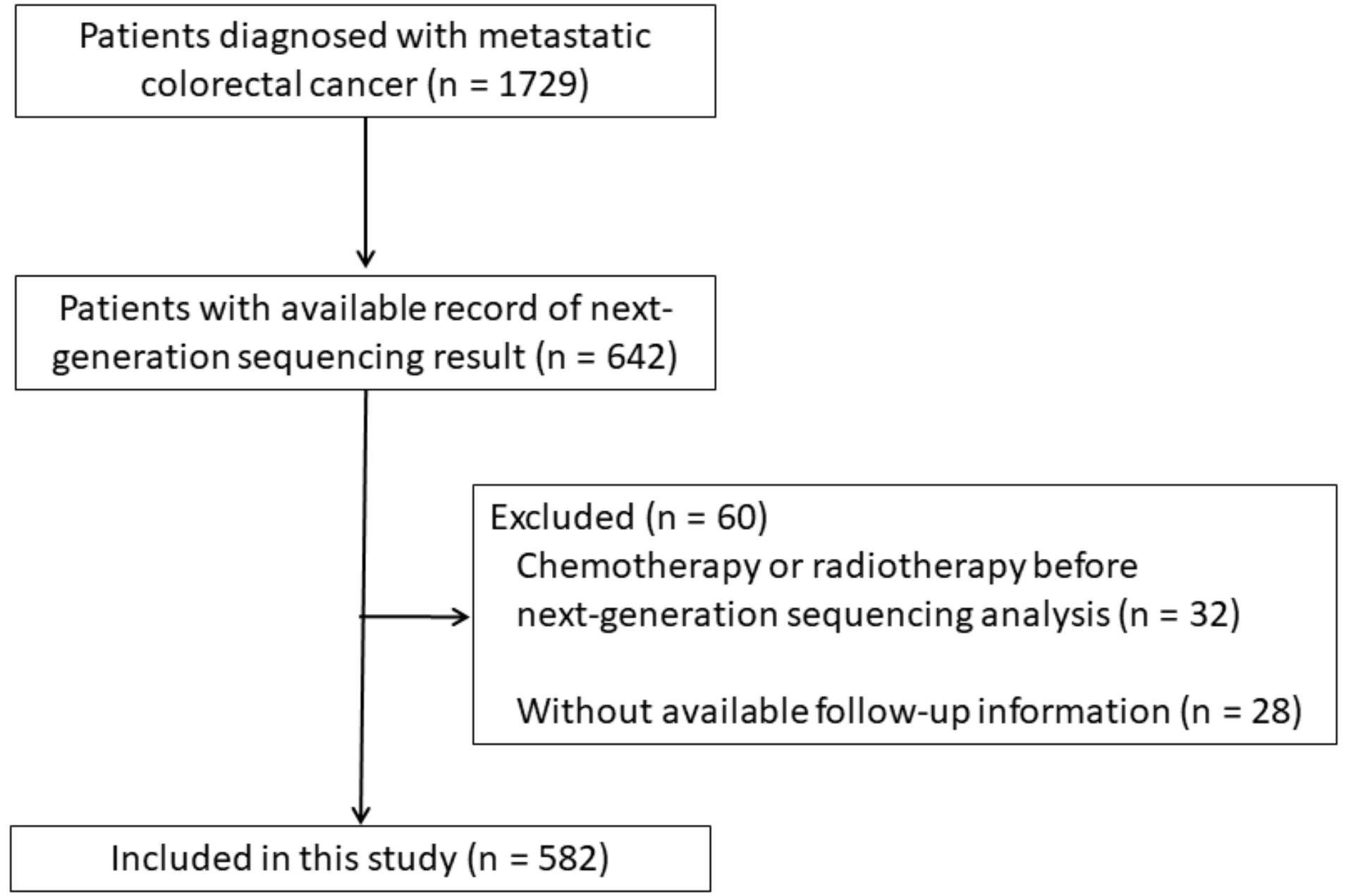

\section{Figure 1}

Flow chart describing patient selection for this study.

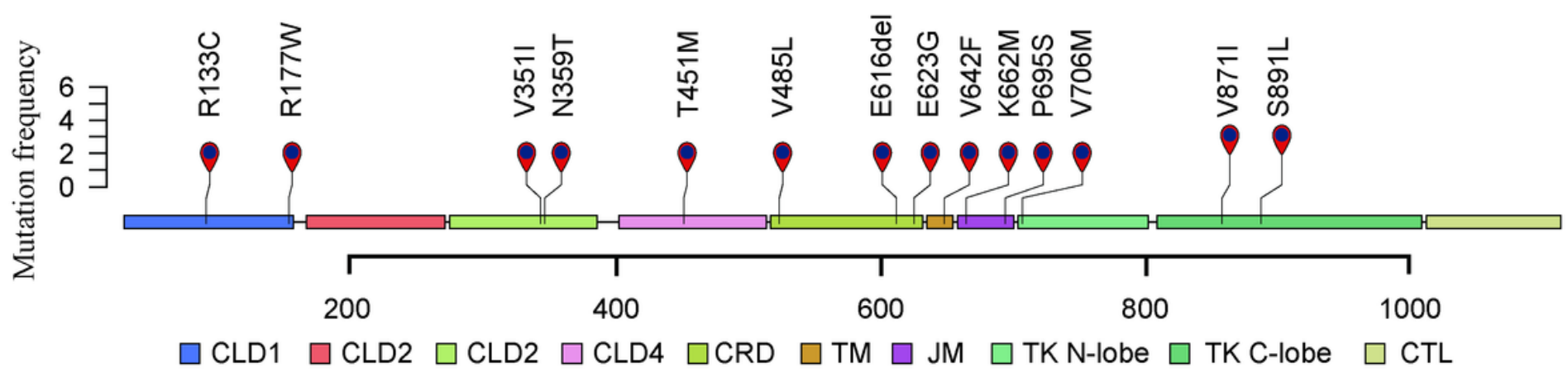

Figure 2

Frequent RET mutations detected in 582 patients with metastatic colorectal cancer. 


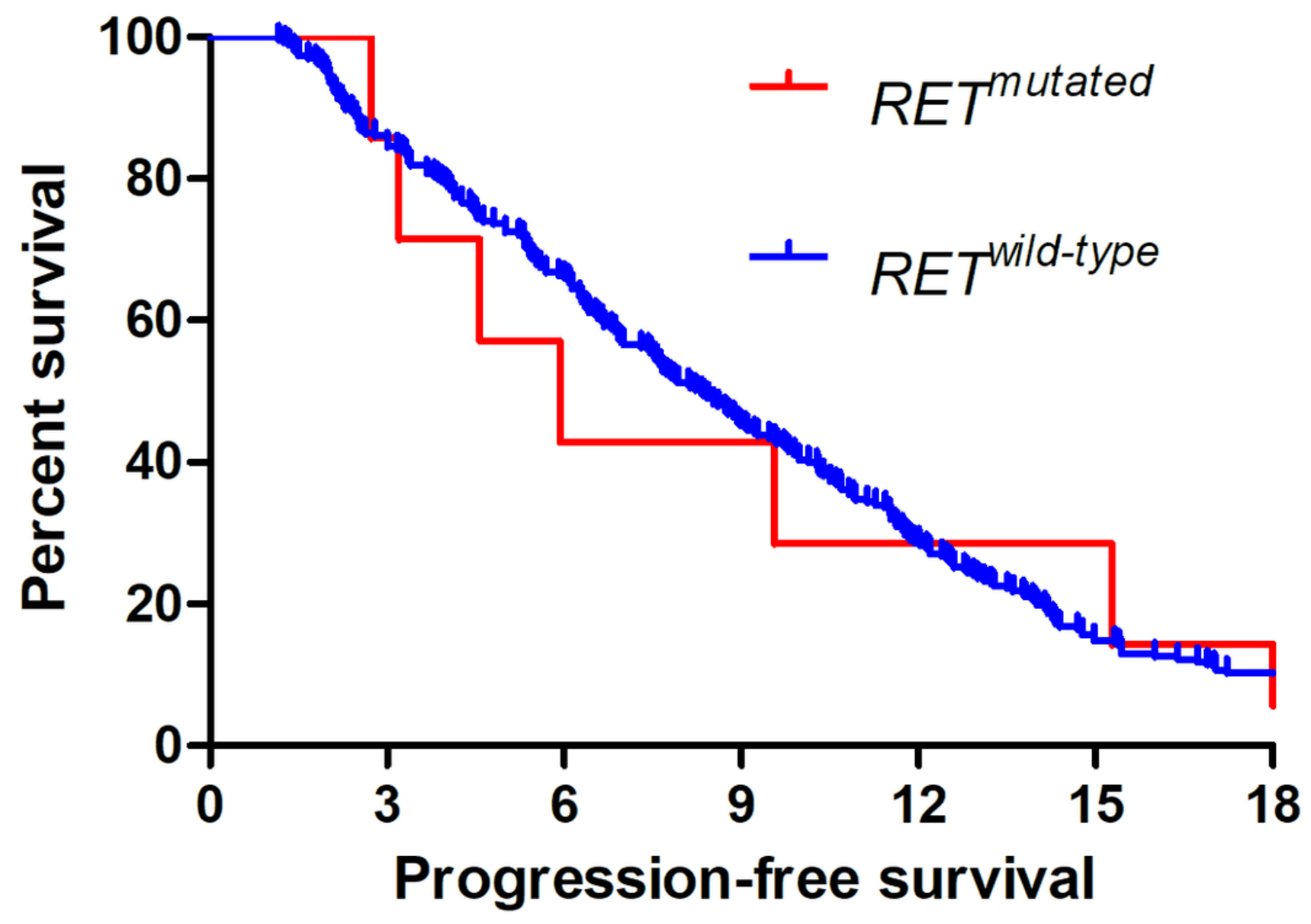

Figure 3

Comparison of progression-free survival between patients with RET wild-type and RET-mutated tumors who were administered oxaliplatin-based first-line chemotherapy.

\section{Supplementary Files}

This is a list of supplementary files associated with this preprint. Click to download.

- supplementarymethod.docx 